Title:

\title{
FIND AND NEUTRALIZE
} CLANDESTINE NUCLEAR WEAPONS

Author(s):

Submitted to:

\section{Los Alamos}

NATIONAL LABORATORY
Gregory H. Canavan, P-DO

RECEIVED

DEC 161997

OSTI

\section{MASTER}

For Report of the Defense Science Board Study of

Transnational Terrorism

Date: September 1997

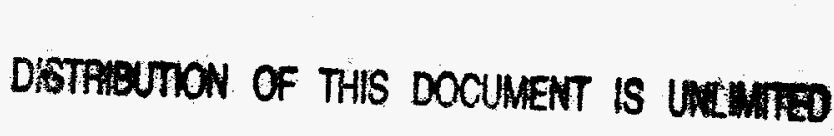
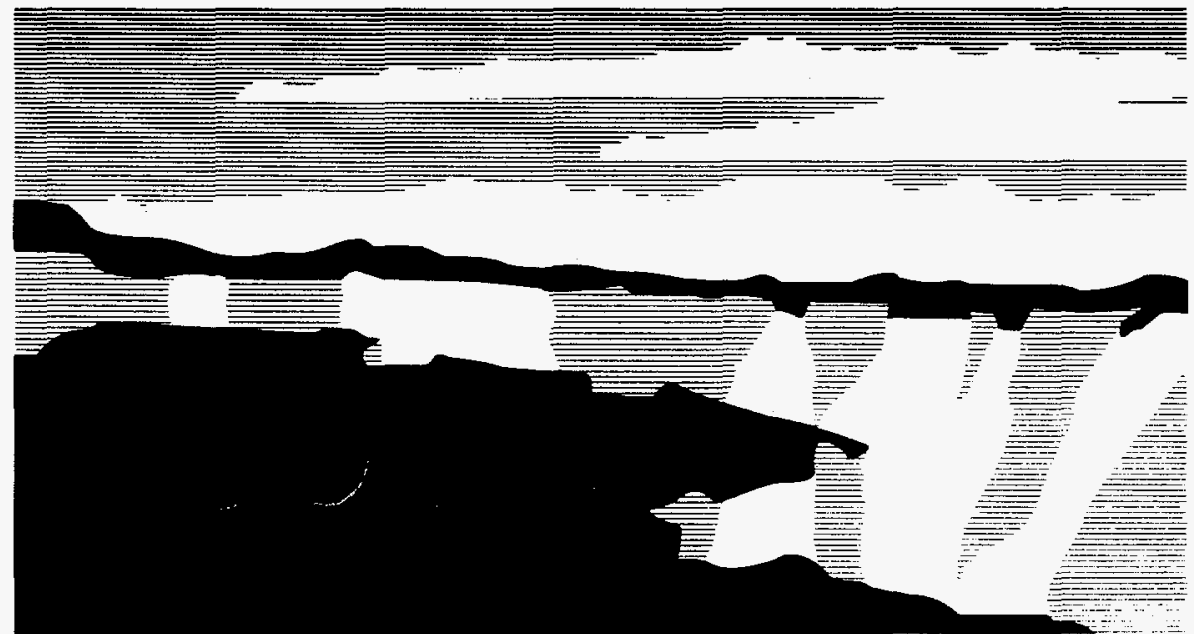

Los Alamos National Laboratory, an affirmative action/equal opportunity employer, is operated by the University of California for the U.S. Department of Energy under contract W-7405-ENG-36. By acceptance of this article, the publisher recognizes that the U.S. Government retains a nonexclusive, royalty-free license to publish or reproduce the published form of this contribution, or to allow others to do so, for U.S. Government purposes. The Los Alamos National Laboratory requests that the publisher identify this article as work performed under the auspices of the U.S. Department of Energy. 


\section{DISCLAIMER}

This report was prepared as an account of work sponsored by an agency of the United States Government. Neither the United States Government nor any agency thereof, nor any of their employees, makes any warranty, express or implied, or assumes any legal liability or responsibility for the accuracy, completeness, or usefulness of any information, apparatus, product, or process disclosed, or represents that its use would not infringe privately owned rights. Reference herein to any specific commercial product, process, or service by trade name, trademark, manufacturer, or otherwise does not necessarily constitute or imply its endorsement, recommendation, or favoring by the United States Government or any agency thereof. The views and opinions of authors expressed herein do not necessarily state or reflect those of the United States Government or any agency thereof. 


\section{DISCLAmizR}

Portions of this docoment migy be illegibie in electronic image products. Imoges are produced from the best arailable origion docomentert 


\section{FIND \& NEUTRALIZE CLANDESTINE NUCLEAR WEAPONS}

\section{What are we trying to do?}

The objective of finding nuclear material at entry portals is to provide a secure perimeter as large as a weapon damage radius so that operations could be conducted within it relatively unencumbered. The objective of wide area search for nuclear material to provide a safe zone of similar dimensions in an area in which it is not possible to maintain a secure perimeter, to provide assurance for civilians living at an area at risk, or to provide rapid, wide area search of regions that could conceal nuclear threats to forces in the field.

This rapid, wide-area, and confident detection of nuclear materials is the essential first step in developing the ability to negate terrorist nuclear assemblies or weapons. The ability to detect and negate nuclear materials are necessary to prevent the forced, massive evacuation of urban populations or the disruption of military operations in response to terrorist threats.

\section{How is it done now?}

Search. Current portals utilize large volume $\left(\sim 4 \times 10^{4} \mathrm{~cm}^{3}\right)$ plastic scintillator which give the highest sensitivity per unit cost. These detectors have very low energy resolution but are effective for the application.

Current wide area search employs man-portable and vehicle-portable radiation detectors to search for radiation sources. These radiation detectors include both gamma and neutron detectors, packaged to be inconspicuous and support a "low profile" search. The man-portable units have a detection range from a few meters to a few tens of meters. The vehicle systems have longer detection ranges, but they also move more rapidly and do not provide a detection range improvement greater than a factor of ten.

Search instruments are based on relative large (few 100 to few $1000 \mathrm{~cm}^{3}$ ) $\mathrm{NaI}(\mathrm{Tl})$ scintillation detectors and on large area (few square meter) moderated ${ }^{3} \mathrm{He}$ proportional counters. These systems are primarily signal to background ratio limited by constraints of size, weight, and collection time. Natural barriers (building walls) of deliberate shielding of the target material further reduce the utility of these systems. Only minor improvements to this basic approach have been seen in twenty five years of development nor are major improvements expected in the future. Solid state detectors such as mercuric iodide or cadmium zinc telluride, while offering improved spectral resolution which reduces the effective background, are currently available only in small $\left(\mathrm{few} \mathrm{cm}^{3}\right)$ sizes which greatly limit the signal. High purity germanium detectors offer high resolution and increased volume (few hundred $\mathrm{cm}^{3}$ ) but results to date have not justified the large cost of these sensors.

The limited range of these detectors makes search a labor-intensive undertaking. Basically, searchers carry the man-portable detectors through the environment, "sweeping" the area for a detection range (predicted for the target device) on either side of their path. For office buildings, hotels, and government 
buildings, the range is usually sufficient to allow the searchers to search effectively from areas of public access such halls or corridors. Using established procedures, the search team covers the building exterior and parking areas first. To speed coverage of parking, the man-portable detectors can be "daisy-chained" to make a detector array and the electronics in a single detector does the signal processing with acquisition times optimized for the speeds and distances involved. Upon starting coverage of the interiors, the search team leader assigns teams to each clearly defined area, usually a floor. The team leader then waits in the security office for reports of radiation detection, special access needs, or other situations requiring his personal attention. He monitors progress and assures safety by constant contact with the teams. Building maintenance or security personnel will assist search teams if the teams require access to areas requiring coverage but not reached from the public access areas. Local law enforcement personnel provide protection. A single search team can cover a single high-rise building $\left(10^{6} \mathrm{ft}^{2}\right)$ in a single eight-hour shift, including initial briefing, transit to the target building, search, recall, and debrief.

Searchers can be deployed from a small professional search cadre or trained from local fire, police, or public safety personnel.

Vehicle searches use modular detector packages that fit into vehicles borrowed or rented at the site of the search. These can include mini-vans for automobile mobility, harbor patrol boats for exterior search of ships and dock areas, and even fork-lift trucks for warehouse searches. Specialized helicoptercarried search equipment is also available, operated by DOE contractors, but this requires low-level flight and is most applicable to search of large open areas. Vehicle search electronics also includes Global Positioning System (GPS) and real-time telemetry of location and radiation alarms.

Areas searched by either vehicles or portable instruments must be maintained in a "clean" state afterward. For this role local law enforcement or building security personnel may be given simple radiation detectors to monitor packages entering a building or vehicles passing through a roadblock. In case of a radiation alarm they can act immediately to secure the source and call for assistance.

Both portable and vehicle searches are monitored from a central office. Where the search deployment is extensive, a Geographical Information System records the coverage, maintaining real-time records of the status of the teams and the areas covered.

Neutralization. The nature of nuclear weapons imposes special considerations on render-safe of these devices not present in conventional bombsquad practice as well as many features having common principles and practices. These considerations are design-dependent, therefore the optimum render-safe must be determined on a case-by-case basis based on the design, protection, and firing set engineering of the actual device encountered. 
The relevant details of the device are determined by "diagnostics", passive or active measurement methods. The render-safe team uses the knowledge gained from these activities to characterize the device and plan to exploit its vulnerabilities. The diagnostic activities can provide all the information required for selection and application of the existing render-safe options, independent of intelligence input.

The available intervention options include a wide range of potential attack methods. There is no one-size-fits-all disablement option; in fact methods which prevent or reduce yield in one case may, in a closely-related device, increase yield or even cause yield where none would have been possible before. Selection of the render-safe option is based on operational priorities:

No nuclear yield

No nuclear material dispersion

No loss of life

No damage to property

Clearly, some situations may not allow the render-safe team to choose an option which fulfills all of these.

Upon selection and approval of a render-safe plan based on the diagnostics obtained by specially-trained technicians, explosive ordnance disposal technicians set up, aim, and remotely operate the render-safe option. Containment structures may be added to prevent dispersal of nuclear material in conventional explosions.

What are the limitations?

Current sensors are based on technology with limited sensitivity, range, and growth potential. They can support portal detection but not useful area search rates. Neutron detectors under development will improve ruggedness but not extend range to levels required for search. Charged particle detectors will never be useful for more than inspection at $\sim 1 \mathrm{~m}$. Photon detectors based on $\mathrm{Ge}$ and high $\mathrm{Z}$ semiconductors are likely to remain small, fragile, and expensive and to require cryogenic cooling for the foreseeable future. Those under development will not provide the ranges required for useful area search.

Current operations assume that the weapon is found for them, accessible, known and relatively user friendly. There is no reason to assume that any of these conditions will be met for terrorist operations. In particular, improved capabilities are needed for area search and to address weapons to which one cannot gain access, which are booby trapped, or which are unfamiliar.

What is the new approach?

A novel approach to nuclear weapon detection is the combination of directional information (imaging) and gamma ray energy ("color") to produce a "gamma ray color camera" (GRCC), which might be able to achieve the few hundred meter ranges needed for effective search.

There are efforts underway to use multiple scatter to infer the directions of neutrons and others to use advanced electronics and detectors to infer the direction of gamma rays, so the concept is not totally novel. The new element is 
the recognition that a sensor consisting of $\sim 10,000$ ten micron plastic sheets, each $\sim 1 \mathrm{~m}$ across, separated by $\sim 0.1 \mathrm{~cm}$ gas gaps containing arrays of $\sim 10$ micron pitch metalized detectors could provide a very compact, efficient, and inexpensive spark chamber ("Nuclear Counter Proliferation with Gamma-Ray Color Camera Technology," 1994). It has been suggested that still simpler designs based on semiconductor technology could suffice for simpler applications (Wood, 1997).

The array would measure the gamma trajectory by detecting the charge from the secondary electrons produced by Compton electron in the gamma scattering. If it is possible to measure the direction of the Compton electron to 1 milliradian, it should be possible to infer the initial energy of the gamma ray to within about $1 \mathrm{keV}$. That would take full advantage of the energy resolution of the detector and produce a comb energy filter with lines about as narrow as those of the gamma rays from the weapon. It would support a energy-optimized range of about $300 \mathrm{~m}$, which would support useful search rates from sensors mounted on trucks or air vehicles (Dickerman and Brackenbush, 1994).

While this approach is promising, acceptable performance from this simple detector array depends on its ability to determine the direction of the Compton electron from the gamma scattering to within $\sim 1$ milliradian, which is comparable to the expected scattering of the Compton electron from a few sheets of detectors. It is argued that centroiding the distribution of secondaries from the Compton electron can reduce this angular error, but that has not been established. It is also argued that using trajectories with many scatterings would "over determine" the gamma trajectory and improve accuracy, although it is not clear how that would come about. For the baseline design above to achieve its desired $300 \mathrm{~m}$ range, it is be necessary to gain about a factor of 5 from both centroiding charge and trajectory over determination. Should either not prove possible, the filter would not achieve the angular and energy resolution required, and the range would degrade an order of magnitude to levels that would not support useful area search (Canavan, 1997).

Given detection, several improved techniques could be used to negate weapons that were not accessible, safe to defuse, or of known design. One is the used of very high velocity explosively driven projectiles. Such projectiles are well developed; their extension to higher velocities is not stressing. If successful, it should produce little or no nuclear yield; however, it is sensitive to uncertainties about the design of the device.

An alternative disablement mechanism, which has been studied less intentionally, is a thermal blanket or microwave source. While the usual disarm procedure is to escalate means as gradually as possible, for many weapons it is possible to surround them in a high temperature bath and boil or bake off the high explosive. This has the nature of a last-chance measure, but a simple one.

These measures assume that the device is detected and addressed on a time scale very long compared to that of firing and fusing, implosion, and yield. In some cases that might not be the case. One might still be searching for the device 
when its detonation sequence is initiated. Even then there is at least one concept that might prevent detonation. It is possible to detect the electromagnetic signature of the weapon's detonators, which is almost unique, at ranges of several $\mathrm{km}$. The weapon could then be localized with differential GPS to $\sim 1 \mathrm{~m}$ at $1 \mathrm{~km} \sim$ $1 \mathrm{mr}$, which is adequate for pointing a particle beam at the weapon to disable it. $\mathrm{A} \sim 0.1 \mathrm{~A}, \mathrm{GeV}$ proton beam could preinitiate the weapon by flooding its pit with neutrons so that it would produce little yield. The approach is robust. It should work for Pu, U235, and weapons of unknown design, so long as they use simple firing systems to achieve $\mathrm{HE}$ initiation and design approaches to criticality.

Why will it be successful?

The gamma ray color camera should be successful because it combines the three most useful features of a weapon: optimal spatial filtering to optimize the point source weapon signal versus the uniform distributed background; optimal energy filtering to optimize the weapon material specific line sources against the diffuse cosmic background; and the use of an uncharged gamma for long propagation converted to a charged Compton for ease of measurement in the detector array. There is some room for degradation in each of these areas.

If all were to work as claimed, the gamma ray color camera would use optimal spatial-color filtering to produce a sensor with high sensitivity, good mobility, and wide area search. It should produce such sensors with simple, inexpensive, fieldable components. The main remaining uncertainties could be removed by modest laboratory demonstrations.

Kinetic energy penetrator disablement should work for many designs because it is largely a matter of achieving a higher velocity than the implosion. There can be little argument over the a thermal blanket technique's technical effectiveness, as the DoD has accidentally "disarmed" weapons this way through accidental fires over the last few decades without nuclear yield. The issue is whether such a capability is needed for inaccessible, unfamiliar weapons.

The detonator detection-beam disablement is less developed. There is little question that the detonator signatures are detectable over several kilometers or that differential GPS could refine that to location measurements of $\sim 1 \mathrm{~m}$. The main issue is the practicality of the beam. The parameters cited above are those of current storage rings, which can be dumped on the time scales cited, with rather better accuracies than those required. Thus, the main issue is not whether such a device could be built or made sufficiently portable for search, it is whether the lack of such a last-ditch search and disablement capability is a serious impediment to civil-military search and neutralization actions.

\section{If successful, what is the payoff?}

The new detector technologies discussed above would permit rapid search at portals, securing of perimeters, and search of large areas for threats to military forces and urban populations. That would eliminate the threat of nuclear materials or weapons in those areas that could otherwise cause widespread confusion, create the possibility of massive damage, and open the way to 
blackmail of civilian and military operations.

The ability to disarm weapons of new or unfamiliar designs would reduce the potential for damage and increase the credibility of assurances to those in those areas. The ability to detect and disarm weapons hidden in the field would reduce or eliminate restrictions on operations and make the occupation of areas accessible to terrorist weapons psychologically feasible in the long term.

\section{References}

G. Canavan, "Gamma Ray Color Camera Performance Issues," Los Alamos National Laboratory report LA-UR-97-3458.

C. Dickerman (ANL) and L. Brackenbush (PNL), "Evaluation of LLNL Gamma Ray Color Camera: RDP Panel Task \#4," Argonne and Pacific Northwest Laboratories report 1994.

"Nuclear Counter Proliferation with Gamma-Ray Color Camera Technology," Lawrence Livermore National Laboratory LLNL Doc.No.PhysBrief 94-004, 7 March 1994.

J. Vitko, R. James, D. Rakestraw, J. Schoeniger, S. Gordon, "Nuclear, Biological, and Chemical Detection Technologies," Sandia National Laboratories White paper, May 1996.

L. Wood, "Silicon-Based Gamma-Ray Color Cameras: High-Performance, Compact, Mass-Producible, and Cheap," Hoover Institution, Stanford University, 26 May 1997. 